Algebraic $\& \mathcal{G}$ Geometric Topology

Volume 1 (2001) 153-172

Published: 21 March 2001

ATG

\title{
Symplectic fillability of tight contact structures on torus bundles
}

\author{
FAN DING \\ HANSJÖRG Geiges
}

\begin{abstract}
We study weak versus strong symplectic fillability of some tight contact structures on torus bundles over the circle. In particular, we prove that almost all of these tight contact structures are weakly, but not strongly symplectically fillable. For the 3 -torus this theorem was established by Eliashberg.
\end{abstract}

AMS Classification 53D35; 57M50, 57R65

Keywords Tight contact structure, weak and strong symplectic filling, contact surgery

\section{Introduction}

A coorientable 2-plane field on an oriented 3-manifold $M$ is called a (positive) contact structure if, for any 1 -form $\alpha$ defining $\xi$ as $\xi=\operatorname{ker} \alpha$, the 3 -form $\alpha \wedge d \alpha$ is a (positive) volume form on $M$. Notice that the sign of $\alpha \wedge d \alpha$ only depends on $\xi$, not on the choice of $\alpha$. In this paper, our contact structures are always understood to be positive. We do not consider non-coorientable contact structures (where the corresponding $\alpha$ only exists locally).

There are various notions of fillability of contact structures, see the survey [3]. The two that we are concerned with in the present paper are weak and strong symplectic fillability. Given a 4 -dimensional symplectic manifold $(W, \omega)$, we orient it by regarding $\omega^{2}$ as a positive volume form. If $W$ has boundary $\partial W$, an orientation of $\partial W$ is defined by the volume form $i_{Y} \omega^{2}$, where $Y$ is any vector field defined along the boundary and pointing outwards. Recall that the condition for a vector field $X$ on a symplectic manifold $(W, \omega)$ to be a Liouville vector field is that $\mathcal{L}_{X} \omega=\omega$. By the Cartan formula for the Lie derivative this may be rewritten as $d\left(i_{X} \omega\right)=\omega$, and this easily implies that $i_{X} \omega$ defines a contact structure on any hypersurface transverse to $X$. 
Definition (a) A contact manifold $(M, \xi)$ is weakly symplectically fillable if $M$ is the boundary of a symplectic manifold $(W, \omega)$ with $\omega \mid \xi$ nondegenerate along $\partial W=M$, and the orientations on $M$ induced by $W$ and $\xi$ agree.

(b) A contact manifold $(M, \xi)$ is strongly symplectically fillable if $M$ is the boundary of a symplectic manifold $(W, \omega)$ admitting a Liouville vector field $X$ near the boundary $\partial W=M$, pointing outwards along $\partial W$, and such that $\xi=\operatorname{ker}\left(i_{X} \omega \mid M\right)$.

Recall that a contact structure $\xi$ on a 3 -manifold $M$ is called overtwisted if there is an embedded 2-disc $D \hookrightarrow M$ such that $\partial D$ is tangent to $\xi$, but $D$ is transverse to $\xi$ along $\partial D$; such a disc is called an overtwisted disc. If no such $D$ exists, then $\xi$ is called tight. Any weakly symplectically fillable contact structure is tight, as was shown by Eliashberg and Gromov, cf. [3].

Clearly strong symplectic fillability implies weak symplectic fillability. The converse was shown to be false by Eliashberg [2]. On the 3 -torus $T^{3}=\mathbf{R}^{3} / \mathbf{Z}^{3}$ with coordinates $(x, y, t)$ and orientation given by $d x \wedge d y \wedge d t$, consider, for non-negative integers $n$, the contact structures $\zeta_{n}$, defined by

$$
\cos (2 \pi(n+1) t) d x-\sin (2 \pi(n+1) t) d y=0 .
$$

The $\zeta_{n}, n \in \mathbf{N}_{0}$, are pairwise nondiffeomorphic and constitute a complete list, up to diffeomorphism, of the tight contact structures on $T^{3}$.

As observed by Giroux [7], the $\zeta_{n}$ are all weakly symplectically fillable. Eliashberg [2] showed that $\zeta_{n}$ is strongly symplectically fillable if and only if $n=0$. Our aim in the present paper is to prove an analogous result for more general $T^{2}$-bundles over $S^{1}$.

We begin with a description of these torus bundles. For each matrix $A \in$ $\mathrm{SL}_{2}(\mathbf{Z})$, let $T_{A}$ denote the quotient of $T^{2} \times \mathbf{R}=\left(\mathbf{R}^{2} / \mathbf{Z}^{2}\right) \times \mathbf{R}$ with coordinates $(\mathbf{x}, t)=\left(\left(\begin{array}{l}x \\ y\end{array}\right), t\right)$ by the transformation $(\mathbf{x}, t) \rightarrow(A \mathbf{x}, t+1)$. We orient $T_{A}$ by the 3 -form $d x \wedge d y \wedge d t$. The $T^{2}$-bundle $T_{A}$ over $S^{1}$ depends, up to diffeomorphism, only on the conjugacy class of $A$ in $\mathrm{SL}_{2}(\mathbf{Z})$. If $A$ is of the form $\left(\begin{array}{ll}1 & 0 \\ k & 1\end{array}\right), k \in \mathbf{Z}$, then we denote the corresponding manifold $T_{A}$ by $T(k)$.

Let $\varphi: \mathbf{R} \rightarrow \mathbf{R}$ be a smooth function whose derivative is strictly positive. The equation

$$
\cos \varphi(t) d x-\sin \varphi(t) d y=0, \quad(x, y, t) \in \mathbf{R}^{3},
$$


defines a contact structure on $\mathbf{R}^{3}$ which we denote by $\widetilde{\zeta}(\varphi)$. For each $\theta \in \mathbf{R}$ let $\Delta_{\theta}$ denote the ray

$$
\left\{\left(\begin{array}{c}
s \sin \theta \\
s \cos \theta
\end{array}\right): s \geq 0\right\} \subset \mathbf{R}^{2} .
$$

If $A\left(\Delta_{\varphi(t)}\right)=\Delta_{\varphi(t+1)}$ for all $t \in \mathbf{R}$, then the contact structure $\widetilde{\zeta}(\varphi)$ on $\mathbf{R}^{3}$ is invariant under the action of the deck transformation group of $T_{A}$ and thus descends to a contact structure on $T_{A}$ which we denote by $\zeta(\varphi)$.

By [8], for each non-negative integer $n$ there exists a smooth function $\varphi: \mathbf{R} \rightarrow$ $\mathbf{R}$ with strictly positive derivative, satisfying $A\left(\Delta_{\varphi(t)}\right)=\Delta_{\varphi(t+1)}$ for all $t \in \mathbf{R}$ and

$$
2 n \pi<\sup _{t \in \mathbf{R}}(\varphi(t+1)-\varphi(t)) \leq 2(n+1) \pi .
$$

Up to fibre preserving isotopy, the contact structure $\zeta(\varphi)$ on $T_{A}$ depends only on $n$. Thus we denote this contact structure simply by $\zeta_{n}$. In [8] it was shown that the $\zeta_{n}$ are tight and pairwise nondiffeomorphic.

The main result of the present paper is the following.

Theorem 1 For each $A \in \mathrm{SL}_{2}(\mathbf{Z})$ and $n \in \mathbf{N}_{0}$ (non-negative integers), the contact manifold $\left(T_{A}, \zeta_{n}\right)$ is weakly symplectically fillable. There exists $n(A) \in$ $\mathbf{N}_{0}$ such that $\left(T_{A}, \zeta_{n}\right)$ is not strongly symplectically fillable for $n>n(A)$.

Combining this with the classification of tight contact structures on $T_{A}^{3}$ due to Giroux [9] and Honda [14], we obtain the following corollary.

Corollary 2 If $A \in S L_{2}(\mathbf{Z})$ with $\operatorname{trace}(A) \neq-2$, then there are only finitely many strongly symplectically fillable contact structures on $T_{A}$ up to diffeomorphism.

Proof By [9, Thm. 1.3], cf. [8, Thm. 6], $T_{A}$ admits only finitely many tight contact structures next to the $\zeta_{n}$, provided that $\operatorname{trace}(A) \neq-2$. In the case $\operatorname{trace}(A)=-2$ there is a further infinite family of tight contact structures.

Additional results for the $T(k)$ are given in Corollary 12 and Proposition 13. 


\section{Preliminaries}

In this section, we review some basic concepts and results needed later. See [6] and [13] for details.

Let $(M, \xi)$ be a contact 3-manifold. Let $\Sigma$ be an orientable surface embedded in $(M, \xi)$. Let $Y$ be the vector field on $\Sigma$ defined by the equation $i_{Y} \Omega=\left.\alpha\right|_{\Sigma}$, where $\alpha$ is a global 1 -form which defines $\xi$, and $\Omega$ is an area form on $\Sigma$. The characteristic foliation $\left.\xi\right|_{\Sigma}$ on $\Sigma$ induced by $\xi$ is the singular foliation represented by $Y$.

A vector field on $M$ is called contact if its flow preserves $\xi$. A closed orientable surface $\Sigma$ embedded in $(M, \xi)$ is called convex if there is a contact vector field $X$ transverse to $\Sigma$. This contact vector field $X$ allows us to find a vertically invariant neighbourhood $\Sigma \times \mathbf{R} \subset M$ of $\Sigma$, where $\Sigma$ is identified with $\Sigma \times\{0\}$. The dividing set $\Gamma_{\Sigma}$ for $X$ is the set of points $x \in \Sigma$ where $X(x) \in \xi(x)$. This dividing set $\Gamma_{\Sigma}$ is a disjoint union of simple closed curves which are transverse to the characteristic foliation $\left.\xi\right|_{\Sigma}$. The isotopy type of $\Gamma_{\Sigma}$ is independent of the choice of $X$. Hence we will slightly abuse notation and call $\Gamma_{\Sigma}$ the dividing set of $\Sigma$. Denote the number of connected components of $\Gamma_{\Sigma}$ by $\# \Gamma_{\Sigma}$.

Let $T$ be a convex torus in a tight contact $3-$ manifold. Then the dividing set $\Gamma_{T}$ consists of an even number $\# \Gamma_{T}$ of parallel essential curves. Fix an identification of $T$ with $\mathbf{R}^{2} / \mathbf{Z}^{2}$. After a diffeomorphism isotopic to the identity, we may assume that the dividing curves are linear. We call the slope of the dividing curves the slope of the convex torus $T$ and denote it by $s(T)$.

Let $V$ be a solid torus. A specified homeomorphism $h: S^{1} \times D^{2} \rightarrow V$ is called a framing of $V$. Fixing such a framing, we identify $\partial V$ with $T^{2}=\mathbf{R}^{2} / \mathbf{Z}^{2}$ by letting $\left\{\left(\begin{array}{c}t \\ 0\end{array}\right): 0 \leq t \leq 1\right\}$ correspond to the meridian of the solid torus $V$, and $\left\{\left(\begin{array}{c}0 \\ t\end{array}\right): 0 \leq t \leq 1\right\}$ correspond to the longitude determined by the framing. With these identifications, the meridian has slope 0 , the longitude slope $\infty$.

The following proposition will prove useful later; see [15, Thm. 8.2] and [13, Prop. 4.3].

Proposition 3 For any integer $k$ (including 0) there exists a unique (up to isotopy fixed at the boundary) tight contact structure on $S^{1} \times D^{2}$ with a fixed convex boundary with $\# \Gamma_{\partial\left(S^{1} \times D^{2}\right)}=2$ and slope $s\left(\partial\left(S^{1} \times D^{2}\right)\right)=1 / k$. 
Remark In [13] this is stated for integers $k$ of a particular sign only. But if slope $1 / k$ can be realised (uniquely), then slope $1 /(k+l)$ can be realised (uniquely) for any integer $l$, since the two slopes are related to each other by an $l$-fold Dehn twist along the meridian, which extends to a diffeomorphism of the solid torus.

Let $F$ be a closed orientable surface and $\mathcal{F}$ be a singular foliation on $F$. Let $\Gamma$ be a disjoint union of simple closed curves embedded in $F$ which are transverse to $\mathcal{F}$. Let $F_{\Gamma}$ denote the compact surface with boundary obtained by cutting $F$ along $\Gamma$. We say that $\Gamma$ divides $\mathcal{F}$ if there is a vector field $Y$ on $F_{\Gamma}$ such that

- $Y$ represents the singular foliation on $F_{\Gamma}$ induced by $\mathcal{F}$;

- $L_{Y} \Omega>0$ for an area form $\Omega$ on $F_{\Gamma}$;

- $Y$ goes outward along $\partial F_{\Gamma}$.

Here is an important result concerning convex surfaces:

Proposition 4 (Giroux [6, Prop. II.3.6]) Let $\Sigma$ be a closed convex surface in a contact 3 -manifold $(M, \xi)$ with contact vector field $X$ and dividing set $\Gamma$ for $X$. If $\mathcal{F}$ is a singular foliation on $\Sigma$ divided by $\Gamma$, then there is an isotopy $\phi_{s}, s \in[0,1]$, of $\Sigma$ such that $\phi_{0}=\mathrm{id}_{\Sigma},\left.\xi\right|_{\phi_{1}(\Sigma)}=\phi_{1}(\mathcal{F})$ and $\phi_{s}(\Sigma)$ is transverse to $X$ for each $s$.

We state two other results, essentially due to Giroux, which will be used in Section 4.

Proposition 5 Let $F$ be a closed orientable surface embedded in a contact 3 -manifold $(M, \xi)$. If $\Gamma$ divides $\left.\xi\right|_{F}$, then $F$ is convex with dividing set $\Gamma$.

This proposition is a consequence of [6], Propositions I.3.4 and II.1.2(b).

Proposition 6 Let $F$ be a compact orientable surface with boundary. Let $\xi_{0}, \xi_{1}$ be two contact structures on $F \times \mathbf{R}$. Let $U$ be a collar neighbourhood of $\partial F$ in $F$. Assume that $\left.\xi_{0}\right|_{F \times\{0\}}$ coincides with $\left.\xi_{1}\right|_{F \times\{0\}}$ and $\xi_{0}=\xi_{1}$ on $U \times(-\epsilon, \epsilon)$, where $\epsilon$ is a positive real number. Then there exist a neighbourhood $V$ of $F \times\{0\}$ in $F \times \mathbf{R}$ and a contact embedding $f:\left(V, \xi_{0}\right) \rightarrow\left(F \times \mathbf{R}, \xi_{1}\right)$ such that $f$ is the identity on $V \cap(U \times(-\epsilon, \epsilon))$ and $f(F \times\{0\})=F \times\{0\}$.

The proof of this proposition is similar to that of [6], Proposition II.1.2(b). 


\section{Contact surgery}

A smooth knot $K: S^{1} \rightarrow M$ in a contact $3-$ manifold $(M, \xi)$ is called Legendrian if its tangent vectors all lie in $\xi$. Any diffeomorphism between Legendrian knots extends to a contactomorphism (i.e. a diffeomorphism preserving contact structures) on some neighbourhoods of the knots. A Legendrian knot $K$ comes equipped with a canonical framing of its normal bundle, which is induced by any vector field transverse to $\xi$, or equivalently, by a vector field in $\xi \mid K$ transverse to $K$. We call this the contact framing of $K$. There is a canonical bijection from (normal) framings of $K$ to the integers $\mathbf{Z}=\pi_{1}(\mathrm{SO}(2))$, given by identifying the contact framing with $0 \in \mathbf{Z}$ and counting right-handed twists positively. Note that for any nullhomologous Legendrian knot $K$, the linking number of $K$ with its push-off determined by framing $k$ is $t b(K)+k$, where $t b(K)$ is the Thurston-Bennequin invariant of $K$.

Rational surgery on $K$ with coefficient $r=p / q \in \mathbf{Q} \cup\{\infty\}$ (with $p, q$ coprime) is defined as follows: Denote a tubular neighbourhood of $\mathrm{K}$ (diffeomorphic to a solid torus) by $\nu K$. Let $(\mu, \lambda)$ be a positively oriented basis for $H_{1}(\partial \nu K ; \mathbf{Z}) \cong$ $\mathbf{Z} \oplus \mathbf{Z}$, where $\lambda$ is determined up to sign as the class of a parallel copy of $K$ determined by the contact framing, and $\mu$ is determined by a suitably oriented meridian (i.e. a nullhomologous circle in $\nu K$ ), cf. [10, p. 672]. We obtain a new manifold $M^{\prime}$ by cutting $\nu K$ out of $M$ and regluing it by a diffeomorphism of $\partial(\nu K)$ sending $\mu$ to $p \mu+q \lambda$. This procedure determines $M^{\prime}$ up to orientationpreserving diffeomorphism.

Consider $N=\mathbf{R}^{2} \times(\mathbf{R} / \mathbf{Z})$ with coordinates $(x, y, z)$ and contact structure $\zeta$ defined by

$$
\cos (2 \pi z) d x-\sin (2 \pi z) d y=0 .
$$

For each $\delta>0$, let

$$
N_{\delta}=\left\{(x, y, z) \in N: x^{2}+y^{2} \leq \delta^{2}\right\} .
$$

We identify $\partial N_{\delta}$ with $\mathbf{R}^{2} / \mathbf{Z}^{2}$, using the contact framing, and write $(\mu, \lambda)$ for a positively oriented basis for $H_{1}\left(\partial N_{\delta} ; \mathbf{Z}\right) \cong \mathbf{Z} \oplus \mathbf{Z}$, with $\mu$ corresponding to a meridian and $\lambda$ to a longitude determined by this framing. A possible representative of $\lambda$ would be

$$
\{(\delta \sin (2 \pi z), \delta \cos (2 \pi z), z): z \in \mathbf{R} / \mathbf{Z}\} .
$$

Note that the vector field $x \frac{\partial}{\partial x}+y \frac{\partial}{\partial y}$ is a contact vector field for $\zeta$ which is transverse to $\partial N_{\delta}$, with dividing set

$$
\Gamma_{\partial N_{\delta}}=\{( \pm \delta \sin (2 \pi z), \pm \delta \cos (2 \pi z), z): z \in \mathbf{R} / \mathbf{Z}\} .
$$


Thus for each $\delta>0$ the torus $\partial N_{\delta}$ is a convex surface with $\# \Gamma_{\partial N_{\delta}}=2$ and $s\left(\partial N_{\delta}\right)=\infty$.

Let $K$ be a Legendrian knot in a contact manifold $(M, \xi)$. Write

$$
C=\{(x, y, z) \in N: x=y=0\}
$$

for the spine of $N$. Then there is a contact embedding $f:\left(N_{2}, \zeta\right) \rightarrow(M, \xi)$ such that $f(C)=K$. We want to construct a contact structure $\xi^{\prime}$ on the manifold $M^{\prime}$ obtained from $M$ by rational surgery on $K$ with coefficient $r=$ $p / q \in \mathbf{Q} \cup\{\infty\}$, where we only consider $r \neq 0$. Let

$$
P=\left\{(x, y, z) \in N: 1 \leq x^{2}+y^{2} \leq 4\right\}=N_{2} \backslash \operatorname{Int} N_{1} .
$$

Let $g: P \rightarrow P$ be an orientation-preserving diffeomorphism sending $\partial N_{\delta}$ to $\partial N_{\delta}, \delta=1,2$, and $\mu$ to $p \mu+q \lambda$. The fact that $p \neq 0$ implies that $\left(g_{*}\right)^{-1}(\zeta)$ is a contact structure on $P$ with respect to which $\partial N_{\delta}$ is a convex torus of non-zero slope. By [13, Thm. 2.3], which gives an enumeration of tight contact structures on the solid torus with convex boundary as in our situation (and in particular shows this set of contact structures to be non-empty), the contact structure $\left(g_{*}\right)^{-1}(\zeta)$ on $P$ can be extended to a tight contact structure $\zeta^{\prime}$ on $N_{2}$. Define

$$
M^{\prime}=\left(M-f\left(N_{1}\right)\right) \cup N_{2} / \sim,
$$

where $x \in P \subset N_{2}$ is identified with $f(g(x)) \in M$. Topologically, $M^{\prime}$ is obtained from $M$ by rational surgery on $K$ with coefficient $r$. It inherits a contact structure $\xi^{\prime}$ from $(M, \xi)$ and $\left(N_{2}, \zeta^{\prime}\right)$. We say that $\left(M^{\prime}, \xi^{\prime}\right)$ is obtained from $(M, \xi)$ by contact $r$-surgery on $K$.

Remark (1) In this construction the assumption $r \neq 0$ is essential. The $g: P \rightarrow P$ corresponding to $p=0, q=1$ leads to a contact structure $\left(g_{*}\right)^{-1}(\zeta)$ on $P$ whose extension to $N_{2}$ (if such exists) is overtwisted; the overtwisted disc being given essentially by a meridianal disc in the solid torus $N_{1}$.

(2) It is not clear a priori that $\left(M^{\prime}, \xi^{\prime}\right)$ is tight, even if $(M, \xi)$ was. In the application of this construction (Proposition 11, in particular) we deal with a situation where one knows two tight contact manifolds $(M, \xi)$ and $\left(M^{\prime}, \xi^{\prime}\right)$ to be contactomorphic outside certain solid tori, and we can conclude there that one is obtained from the other by contact surgery as described.

(3) By analysing the framing conditions in the surgery theorems of [1] and [18], cf. [10, Thm. 1.3] and [4], one sees that contact (-1)-surgery corresponds to a symplectic handlebody construction. In particular, if $\left(M^{\prime}, \xi^{\prime}\right)$ is obtained from a closed contact manifold $(M, \xi)$ by contact $(-1)$-surgery and $(M, \xi)$ 
is strongly symplectically fillable, then $\left(M^{\prime}, \xi^{\prime}\right)$ is also strongly symplectically fillable. Given a Legendrian knot, one can add left-twists to its contact framing by performing a suitable isotopy (non-contact and $C^{0}$-small). That way one can realise topological surgeries with negative integer framing (relative to a given contact framing) as 'handlebody' surgeries. Adding positive twists is not, in general, possible, unless the contact structure is overtwisted. We are mostly concerned with contact $(1 / k)$-surgeries, $k \in \mathbf{Z} \backslash\{0\}$, which do not correspond to a handlebody construction unless $1 / k=-1$.

Proposition 7 If $r=1 / k$, where $k$ is an integer, then, up to contactomorphism, the contact manifold $\left(M^{\prime}, \xi^{\prime}\right)$ depends only on $r$ (and $(M, \xi)$ and $K$, of course). That is, it is independent of the choices of $f, g$ and $\zeta^{\prime}$.

Proof The scaling map $(x, y, z) \mapsto(s x, s y, z)$ defines a contactomorphism $\left(N_{\delta}, \zeta\right) \rightarrow\left(N_{s \delta}, \zeta\right)$. Hence, given two contact embeddings $f_{i}:\left(N_{2}, \zeta\right) \rightarrow(M, \xi)$, $i=1,2$, we can compare either with a third such embedding that maps $N_{2}$ into the interior of $f_{i}\left(N_{1}\right)$.

We may therefore assume that $K=C \subset N$, the contact embedding $f_{1}$ is the inclusion map $N_{2} \subset N$, and the contact embedding $f=f_{2}$ sends $N_{2}$ into the interior Int $N_{1}$ of $N_{1}$.

Note that if $r=1 / k$, then the diffeomorphism $g$ may be assumed to have the following effect on $\mu$ and $\lambda$, since $g \mid \partial N_{\delta}$ is determined up to isotopy by its action on homology, corresponding to an element of $\mathrm{SL}_{2}(\mathbf{Z})$ :

$$
\mu \longmapsto \mu+k \lambda, \quad \lambda \longmapsto \lambda-l(\mu+k \lambda),
$$

where $l$ is some integer. (Different choices of $l$ correspond to Dehn twists along a meridian of the solid torus that is glued back; these Dehn twists extend to diffeomorphisms of the solid torus and hence have no topological effect.) Then $g^{-1}$ sends $\lambda$ to $l \mu+\lambda$. This implies that as we pull back $\zeta$ to $\left(g_{*}\right)^{-1} \zeta$, we obtain a contact structure on $N_{2} \backslash \operatorname{Int}\left(N_{1}\right)$ with $\# \Gamma_{\partial N_{2}}=2$ and $s\left(\partial N_{2}\right)=1 / l$. So by Proposition 3 the extension of $\left(g_{*}\right)^{-1} \zeta$ to a tight contact structure $\zeta^{\prime}$ on the copy of $N_{2}$ to be glued back is unique.

Let $\left(M_{1}^{\prime}, \xi_{1}^{\prime}\right)$ be the contact manifold obtained from $N_{2}$ by contact $r$-surgery along $C \subset N_{2}$ using the inclusion $N_{2} \subset N_{2}$, and let $\left(M_{2}^{\prime}, \xi_{2}^{\prime}\right)$ be the contact manifold obtained similarly using the contact embedding $f: N_{2} \rightarrow \operatorname{Int} N_{1} \subset$ $N_{2}$.

By what we have just observed, the tight contact structure $\xi_{1}^{\prime}$ is uniquely determined by the fact that it coincides with $\zeta$ near $\partial N_{2}=\partial M_{1}^{\prime}$. We also know 
that $\xi_{2}^{\prime}$ coincides with $\zeta$ outside $f\left(N_{1}\right)$, and the manifolds $M_{1}^{\prime}$ and $M_{2}^{\prime}$ are diffeomorphic under a diffeomorphism that is the identity near $\partial M_{1}^{\prime}=\partial M_{2}^{\prime}$.

By the definition of contact $r$-surgery, the contact manifold

$$
\left(M_{2}^{\prime} \backslash\left(N_{2} \backslash f\left(N_{2}\right)\right), \xi_{2}^{\prime}\right)
$$

is tight. It now suffices to show that $\left(M_{2}^{\prime}, \xi_{2}^{\prime}\right)$ is tight, because we then know that it is completely determined by its boundary data, which coincide with those of $\left(M_{1}^{\prime}, \xi_{1}^{\prime}\right)$.

Recall that if a contact structure $\xi$ on a manifold $M$ is written as the kernel of a 1 -form $\alpha$, there is a one-to-one correspondence between contact vector fields $X$ and functions on $M$ given by $X \mapsto \alpha(X)$, cf. [16]. The function $H=\alpha(X)$ is called the Hamiltonian function corresponding to $X$.

So the contact vector field $X=-\left(x \frac{\partial}{\partial x}+y \frac{\partial}{\partial y}\right)$ for $\zeta$ on $N_{2}$ corresponds in this way to some Hamiltonian function. By multiplying this function with a bump function that is identically 1 on $N_{1}$ and identically zero near $\partial N_{2}$ we can construct a contactomorphism $N_{2} \rightarrow N_{2}$ that is the identity near $\partial N_{2}$ and sends $N_{1}$ into $N_{\delta}$ for any given $\delta>0$. By precomposing $f$ with such a diffeomorphism, we may assume that

$$
f\left(N_{1}\right) \subset \operatorname{Int} N_{\delta}, \quad N_{\delta} \subset \operatorname{Int} f\left(N_{2}\right)
$$

for a suitable $\delta>0$.

By multiplying the Hamiltonian function of $X$ with a bump function that is identically 0 on $f\left(N_{1}\right)$ and identically 1 outside $N_{\delta}$, we get a Hamiltonian function defined also on $M_{2}^{\prime}$ whose contact flow will ultimately move $N_{2}$ into $f\left(N_{2}\right)$. So this will define a contact embedding

$$
\left(M_{2}^{\prime}, \xi_{2}^{\prime}\right) \hookrightarrow\left(M_{2}^{\prime} \backslash\left(N_{2} \backslash f\left(N_{2}\right)\right), \xi_{2}^{\prime}\right) .
$$

This completes the proof of the proposition.

Proposition 8 If $\left(M^{\prime}, \xi^{\prime}\right)$ is obtained from $(M, \xi)$ by contact $(1 / k)$-surgery, then $(M, \xi)$ is obtained from $\left(M^{\prime}, \xi^{\prime}\right)$ by contact $(-1 / k)$-surgery.

Proof By the preceding proposition it suffices to consider the following situation: Let $\left(M^{\prime}, \xi^{\prime}\right)$ be the manifold obtained from $(N, \zeta)$ by contact $(1 / k)-$ surgery along $C \subset N$, using the inclusion $N_{2} \subset N$. Let $(M, \xi)$ be the manifold obtained from $\left(M^{\prime}, \xi^{\prime}\right)$ by contact $(-1 / k)$-surgery along a spine of the solid torus $N_{1}$ that was attached to $N \backslash N_{1}$ to form $M^{\prime}$. We want to show that $(M, \xi)$ is contactomorphic to $(N, \zeta)$. 
We can obtain $\left(M^{\prime}, \xi^{\prime}\right)$ by gluing $N_{2}$ to $N \backslash N_{1}$ using the attaching map $g: P \rightarrow$ $P$ described by

$$
\mu \longmapsto \mu+k \lambda, \quad \lambda \longmapsto \lambda,
$$

and then extending $\left(g_{*}\right)^{-1} \zeta$ over $N_{2}$ to a unique tight contact structure $\zeta^{\prime}$. We observed in the proof of the preceding proposition that the torus $\partial N_{1}$ in $\left(N_{2}, \zeta^{\prime}\right)$ is a convex surface with $\# \Gamma_{\partial N_{1}}=2$ and $s\left(\partial N_{1}\right)=\infty$.

By Propositions 3 and 4 and arguments similar to those in the preceding proof, we can find a contact embedding $\left(N_{2}, \zeta\right) \hookrightarrow\left(N_{2}, \zeta^{\prime}\right)$ isotopic to the identity and sending $P$ into $P$. Now perform the $(-1 / k)$-contact surgery on $\left(M^{\prime}, \xi^{\prime}\right)$ using this embedding (composed with $g$ ), and call the resulting contact manifold $(M, \xi)$. The gluing for this surgery may be described by

$$
\mu \longmapsto \mu-k \lambda, \quad \lambda \longmapsto \lambda .
$$

It is a straightforward check that the topological effect of this second surgery is to cancel the first surgery, because the composition of these maps sends $\mu$ to $\mu$ (in fact, it is the identity map). A further application of Proposition 3 shows that $(M, \xi)$ is indeed contactomorphic to $(N, \zeta)$.

Let $\left(M^{\prime}, \xi^{\prime}\right)$ be obtained from $(M, \xi)$ by contact $(1 / n)$-surgery on a Legendrian knot $K$, where $n>1$. Let $\left(M^{\prime \prime}, \xi^{\prime \prime}\right)$ be obtained from $(M, \xi)$ by contact $(1 /(n-1))$-surgery on the same knot $K$. By the same methods as in the proof of the preceding proposition one sees that $\left(M^{\prime}, \xi^{\prime}\right)$ can be obtained from $\left(M^{\prime \prime}, \xi^{\prime \prime}\right)$ by contact $(+1)$-surgery. Similarly, contact $(1 / n)$-surgery with $n<-1$ can be realised as a contact $(1 /(n+1))$-surgery followed by a contact $(-1)$-surgery. Thus, by induction we have:

Proposition 9 If $\left(M^{\prime}, \xi^{\prime}\right)$ is obtained from $(M, \xi)$ by contact $(1 / n)$-surgery, $n \neq 0$, it may also be obtained by $|n|$ times contact $\varepsilon$-surgery, where $\varepsilon=$ $n /|n|=\operatorname{sign}(n) \in\{-1,1\}$.

Combined with remark (3) above, the two preceding propositions yield the following result.

Proposition 10 Let $(M, \xi)$ be a closed contact $3-$ manifold. Let $n$ be a positive integer. If $\left(M^{\prime}, \xi^{\prime}\right)$ is obtained from $(M, \xi)$ by contact $(-1 / n)$-surgery and $(M, \xi)$ is strongly symplectically fillable, then $\left(M^{\prime}, \xi^{\prime}\right)$ is strongly symplectically fillable. If $\left(M^{\prime}, \xi^{\prime}\right)$ is obtained from $(M, \xi)$ by contact $(1 / n)$-surgery and $(M, \xi)$ is not strongly symplectically fillable, then $\left(M^{\prime}, \xi^{\prime}\right)$ is not strongly symplectically fillable. 


\section{Proof of the main result}

The key step in the proof of Theorem 1 is contained in the following proposition.

Proposition 11 Let $A_{0} \in \mathrm{SL}_{2}(\mathbf{Z})$, let $E_{k}=\left(\begin{array}{cc}1 & 0 \\ k & 1\end{array}\right), k \in \mathbf{Z} \backslash\{0\}$, and let $n_{0}$ be a positive integer. Then contact $(-1 / k)$-surgery on $\left(T_{A_{0}}, \zeta_{n_{0}}\right)$ yields $\left(T_{A}, \zeta_{n}\right)$, where $A=E_{k} A_{0}$ and $n \in\left\{n_{0}, n_{0}-1\right\}$ for $k>0$, or $n \in\left\{n_{0}, n_{0}+1\right\}$ for $k<0$. If $A_{0}$ is of type $E_{l}$, then $n$ is determined explicitly as follows.

\begin{tabular}{c|l|c}
\multicolumn{1}{c|}{$k$} & $A_{0}$ & $n$ \\
\hline$>0$ & $E_{l}, l<-k$ or $l \geq 0$ & $n_{0}$ \\
& $E_{l},-k \leq l<0$ & $n_{0}-1$ \\
$<0$ & $E_{l}, l \geq-k$ or $l<0$ & $n_{0}$ \\
& $E_{l}, 0 \leq l<-k$ & $n_{0}+1$
\end{tabular}

Remark An exact determination of the value of $n$ corresponding to any given $A_{0}$ is feasible and would allow an estimate on the bound $n(A)$ in Theorem 1.

Using this proposition, we can formulate a strengthening of Theorem 1 in the case $A=E_{k}, k<0$.

Corollary 12 The contact manifold $\left(T(k), \zeta_{n}\right)$ is not strongly symplectically fillable for $k \leq 0$ and $n \geq 2$.

Proof For $k=0$, that is, $T(0)=T^{3}$, this is the result of Eliashberg mentioned in the introduction, which holds true even for $n=1$. By the preceding proposition, $\left(T(k), \zeta_{n+1}\right), k<0$, is obtained from $\left(T^{3}, \zeta_{n}\right)$ by contact $(-1 / k)$-surgery. The result now follows from Proposition 10.

Here is a complementary result.

Proposition 13 The contact manifold $\left(T(k), \zeta_{0}\right)$ is strongly symplectically fillable for all $k \in \mathbf{Z}$.

Proof For $k=0$ this is well-known, see [2]. For positive $k$ it is a consequence of Propositions 10 and 11 (which holds true also for $n_{0}=0$ and $A_{0}=E_{0}$ ).

For negative $k$ we use a construction analogous to [17, Lemma 2.6]. Let $\varphi: \mathbf{R} \rightarrow$ $\mathbf{R}$ be a smooth function with strictly positive derivative, $E_{k}\left(\Delta_{\varphi(t)}\right)=\Delta_{\varphi(t+1)}$ 
for all $t \in \mathbf{R}$, and $\varphi(0)=\pi / 2$. Notice that $k<0$ then implies $0<\varphi(t)<\pi$ for all $t \in \mathbf{R}$. So we may define $\zeta_{0}$ as kernel of the contact form $\beta=d y-\cot \varphi(t) d x$ (defined on $T(k)$ ).

Projection onto the $x$ - and $t$-coordinate gives $T(k)$ the structure of a principal $S^{1}$-bundle $\pi: T(k) \rightarrow T^{2}$. Let $L$ be the associated complex line bundle $T(k) \times_{S^{1}} \mathbf{C}$, and write $L_{0}$ for its zero section. Write $\theta$ for the angular coordinate and $r$ for the radial coordinate in the $\mathbf{C}$-fibre, so that $\frac{\partial}{\partial y}=\frac{\partial}{\partial \theta}$ on $T(k) \subset L$. The vector fields $\frac{\partial}{\partial \theta}$ and $\frac{\partial}{\partial r}$ are defined on $L \backslash L_{0}$, and $\beta$ extends to an $S^{1}$-invariant 1 -form on $L \backslash L_{0}$ satisfying $\beta\left(\frac{\partial}{\partial \theta}\right)=1$ and $\beta\left(\frac{\partial}{\partial r}\right)=0$. We then have

$$
d \beta=\pi^{*}\left(\varphi^{\prime}(t) \csc ^{2} \varphi(t) d t \wedge d x\right)
$$

Set

$$
\omega=d\left(\left(r^{2}+1\right) \beta\right)=\left(r^{2}+1\right) d \beta+2 r d r \wedge d \beta .
$$

It is a straightforward check that $\omega$ is a symplectic form defined on all of $L$, and that $X=\frac{r^{2}+1}{2 r} \frac{\partial}{\partial r}$ is a Liouville vector field for $\omega$ defined on $L \backslash L_{0}$, and $i_{X} \omega=\left(r^{2}+1\right) \beta$. So the unit disc bundle $T(k) \times{ }_{S^{1}} D^{2}$ gives a strong symplectic filling of $\left(T(k), \zeta_{0}\right)$ for $k<0$.

Assuming Proposition 11, we can now prove the part of Theorem 1 concerned with strong symplectic fillability.

Proposition 14 For each $A \in \mathrm{SL}_{2}(\mathbf{Z})$ there exists an $n(A) \in \mathbf{N}_{0}$ such that $\left(T_{A}, \zeta_{n}\right)$ is not strongly symplectically fillable for $n>n(A)$.

Proof It is well-known (and easy to prove) that $\mathrm{SL}_{2}(\mathbf{Z})$ is generated by $E_{-1}$ and $E_{1}^{\prime}=\left(\begin{array}{ll}1 & 1 \\ 0 & 1\end{array}\right)$. Moreover, the product $E_{-1} E_{1}^{\prime}$ is of order 6 in $\mathrm{SL}_{2}(\mathbf{Z})$, which implies that $E_{-1}^{-1}$ and $\left(E_{1}^{\prime}\right)^{-1}$ can be expressed as a product in $E_{-1}$ and $E_{1}^{\prime}$. Thus, with $A \in \mathrm{SL}_{2}(\mathbf{Z})$ given, we may write it in the form $A=A_{1} \cdots A_{m}$ with $m \in \mathbf{N}$ and $A_{i} \in\left\{E_{-1}, E_{1}^{\prime}\right\}$. Set $A_{m}^{\prime}=E_{0}$ and $A_{i}^{\prime}=A_{i+1} \cdots A_{m}$ for $i=1, \ldots, m-1$, so that $A_{i-1}^{\prime}=A_{i} A_{i}^{\prime}$.

If $A_{i}=E_{-1}$, then by Proposition 11 we know that $\left(T_{A_{i-1}^{\prime}}, \zeta_{n}\right)$ is obtained from $\left(T_{A_{i}^{\prime}}, \zeta_{n_{0}}\right)$ by contact $(+1)$-surgery, where $n \in\left\{n_{0}, n_{0}+1\right\}$ is chosen suitably.

If $A_{i}=E_{1}^{\prime}$, we observe that with $B=\left(\begin{array}{cc}0 & 1 \\ -1 & 0\end{array}\right)$ we can write

$$
A_{i-1}^{\prime}=A_{i} A_{i}^{\prime}=B\left(E_{-1}\left(B^{-1} A_{i}^{\prime} B\right)\right) B^{-1} .
$$


Since conjugate matrices $B_{0}$ and $B_{1}=B B_{0} B^{-1}$ give rise to contactomorphic torus bundles $\left(T_{B_{0}}, \zeta_{n}\right)$ and $\left(T_{B_{1}}, \zeta_{n}\right)$, we conclude once again that $\left(T_{A_{i-1}^{\prime}}, \zeta_{n}\right)$ is obtained from $\left(T_{A_{i}^{\prime}}, \zeta_{n_{0}}\right)$ by contact $(+1)$-surgery for a suitable $n \in\left\{n_{0}, n_{0}+1\right\}$.

By induction, there exists $n(A) \in \mathbf{N}_{0}$ such that $\left(T_{A}, \zeta_{n+n(A)}\right)$ is obtained from $\left(T^{3}, \zeta_{n}\right)$ by $m$ times contact $(+1)$-surgery. Thus, Eliashberg's theorem and Proposition 10 imply that $\left(T_{A}, \zeta_{n+n(A)}\right)$ is not strongly symplectically fillable for $n \geq 1$.

To prove the part of Theorem 1 concerned with weak symplectic fillability, we first make the following observation.

Proposition 15 For each $A \in \mathrm{SL}_{2}(\mathbf{Z})$ there exists a compact symplectic manifold $(W, \omega)$ such that $T_{A}$ is the oriented boundary of $W$ and $\omega$ is nondegenerate on each torus fibre of $T_{A}$.

Proof Given $A \in \mathrm{SL}_{2}(\mathbf{Z})$, we write it in the form $A=A_{1} \cdots A_{m}$ with each $A_{i}$ equal to $E_{-1}$ or $E_{1}^{\prime}$. Let $\pi: S \rightarrow \mathbf{C} P^{1}$ be a nodal elliptic surface with a section, without multiple fibres, and with Euler number (or number of singular fibres) equal to $12 d \geq 2 m$. For the existence of such a surface see [5, p. 64]. This surface is algebraic [5, p. 34] and thus Kähler; in particular we find a symplectic from $\omega$ on $S$ that restricts to an area form on each nonsingular fibre (since these are complex submanifolds).

By the arguments in Section 2.3 of [5] we find a simple closed loop $\gamma$ in $\mathbf{C} P^{1}$ along which the monodromy of the fibration $\pi$ equals $A$. Let $D \subset \mathbf{C} P^{1}$ be the disc whose oriented boundary is $\gamma$. Then $\left(W=\pi^{-1}(D), \omega\right)$ is the desired symplectic manifold.

Here is an alternative and slightly more direct argument: Observe that $E_{-1}$ and $E_{1}^{\prime}$ correspond to positive Dehn twists of $T^{2}$. This implies that there is an orientable Lefschetz fibration $W \rightarrow D^{2}$ with generic fibre a torus, $m$ singular fibres, and monodromy along $\partial D^{2}$ equal to $A$, cf. [11, Section 8.2]. Such a Lefschetz fibration admits a symplectic form $\omega$ with the described properties, see [11, Thm. 10.2.18]. Since the base of the fibration is $D^{2}$, the second homology group of the total space is generated by the fundamental class of the fibre (this remains true in the presence of singular fibres). So the homological condition in the cited theorem, necessary to apply Thurston's symplectic fibration construction, is trivially satisfied.

Here is the part of Theorem 1 concerned with weak symplectic fillability: 
Proposition 16 For each $A \in \mathrm{SL}_{2}(\mathbf{Z})$ and $n \in \mathbf{N}_{0}$, the contact manifold $\left(T_{A}, \zeta_{n}\right)$ is weakly symplectically fillable.

Proof Represent $\zeta_{n}$ by $\zeta(\varphi)$, i.e.

$$
\cos \varphi(t) d x-\sin \varphi(t) d y=0,
$$

where $\varphi$ is as described in the introduction. The properties of $\varphi$ imply that we can find a smooth function $\lambda: \mathbf{R} \rightarrow \mathbf{R}^{+}$such that the contact 1 -form

$$
\alpha=\lambda(t)(\cos \varphi(t) d x-\sin \varphi(t) d y)
$$

is invariant under the transformation $(\mathbf{x}, t) \mapsto(A \mathbf{x}, t+1)$ and thus descends to a contact form (which we continue to denote $\alpha$ ) on $T_{A}$ representing $\zeta_{n}$.

Observe that the 1 -form $\alpha_{\varepsilon}=(1-\varepsilon) d t+\varepsilon \alpha$ is a contact form for any $\varepsilon \in(0,1]$, and in view of the well-known Gray stability theorem [12] it defines a contact structure equivalent to $\zeta_{n}$. For $\varepsilon \searrow 0$ the contact planes ker $\alpha_{\varepsilon}$ approach the tangent spaces along the fibres of $T_{A}$. Hence, the symplectic form $\omega$ on $W$ constructed in the preceding proposition will have the property that $\omega \mid \operatorname{ker} \alpha_{\varepsilon}$ is nondegenerate for $\varepsilon>0$ sufficiently small.

To complete the proof of our main theorem it remains to prove Proposition 11. We only do this for the case $k=1$; the other cases are analogous.

Let $A_{0} \in \mathrm{SL}_{2}(\mathbf{Z})$ be given and set $A=E_{1} A_{0}$. Let $\varphi_{0}: \mathbf{R} \rightarrow \mathbf{R}$ be a smooth function with strictly positive derivative, and satisfying $A\left(\Delta_{\varphi_{0}(t)}\right)=\Delta_{\varphi_{0}(t+1)}$, where $\Delta_{\theta}$ was defined in the introduction. The non-negative integer $n_{0}$ determined by

$$
2 n_{0} \pi<\sup _{t \in \mathbf{R}}\left(\varphi_{0}(t+1)-\varphi_{0}(t)\right) \leq 2\left(n_{0}+1\right) \pi
$$

will be referred to as the twisting of $\varphi_{0}$. Assume in addition that $\varphi_{0}(0)=0$.

Lemma 17 There is a smooth function $\varphi: \mathbf{R} \rightarrow \mathbf{R}$ with strictly positive derivative, satisfying $A\left(\Delta_{\varphi(t)}\right)=\Delta_{\varphi(t+1)}$, as well as $\varphi(0)=\varphi_{0}(0)=0$ and $\varphi(-1)=\varphi_{0}(-1)$. The twisting $n$ of this function $\varphi$ depends on $A_{0}$ and $n_{0}$ as described in Proposition 11.

Proof It is possible to choose the values of $\varphi(t)$ equal to those of $\varphi_{0}(t)$ at $t=0$ and $t=-1$ and still satisfy the appropriate equivariance condition because $\left(\begin{array}{l}0 \\ 1\end{array}\right)=\left(\begin{array}{c}\sin \varphi_{0}(0) \\ \cos \varphi_{0}(0)\end{array}\right)$ is an eigenvector of $E_{1}$ with eigenvalue 1 . 
(i) First consider the case that $\left(\begin{array}{l}0 \\ 1\end{array}\right)$ is an eigenvector of $A_{0}$ with positive eigenvalue. This is equivalent to saying that $A_{0}$ is of type $E_{l}$. A straightforward analysis shows that in this case

$$
\varphi_{0}(-1)= \begin{cases}-2 n_{0} \pi & \text { if } l<0 \\ -2\left(n_{0}+1\right) \pi & \text { if } l \geq 0\end{cases}
$$

The same analysis applies to $A=E_{1} A_{0}=E_{l+1}$. That is, the function $\varphi$ with the described properties has twisting $n$ determined by

$$
\varphi(-1)= \begin{cases}-2 n \pi & \text { if } l+1<0, \\ -2(n+1) \pi & \text { if } l+1 \geq 0 .\end{cases}
$$

Since $\varphi(-1)=\varphi_{0}(-1)$ by assumption, we have $n=n_{0}$ for $l \neq-1$, and $n=n_{0}-1$ for $l=-1$.

(ii) Now assume that $A_{0}$ is not of type $E_{l}$. Then $\varphi_{0}(-1) \notin 2 \pi \mathbf{Z}$, and one verifies that the twisting $n_{0}$ of $\varphi_{0}$ is determined by

$$
2 n_{0} \pi<\sup _{t \in[-1,0]}\left(\varphi_{0}(t+1)-\varphi_{0}(t)\right) \leq 2\left(n_{0}+1\right) \pi,
$$

cf. [8, p. 791]. Let $\bar{h}: S^{1} \rightarrow S^{1}$ (with $S^{1}=\mathbf{R} / 2 \pi \mathbf{Z}$ ) be the smooth function defined by $E_{1}\left(\Delta_{\theta}\right)=\Delta_{\bar{h}(\theta)}$, and let $h: \mathbf{R} \rightarrow \mathbf{R}$ be the lift of $\bar{h}$ with $h(0)=0$. One checks that $h$ is strictly increasing and $t-\pi / 2 \leq h(t) \leq t$ for all $t \in \mathbf{R}$, with equality $h(t)=t$ for $t \in \pi \mathbf{Z}$.

The required function $\varphi$ can be defined by smoothing the function

$$
\varphi(t)= \begin{cases}\varphi_{0}(t) & -3 / 4 \leq t \leq 0 \\ h\left(\varphi_{0}(t)\right) & 0 \leq t \leq 3 / 4\end{cases}
$$

at $t=0$, and then extending it to all $t \in \mathbf{R}$ by imposing the appropriate equivariance property.

Since the smoothing is done at $t=0$, and $\left(\begin{array}{c}\sin \varphi_{0}(0) \\ \cos \varphi_{0}(0)\end{array}\right)=\left(\begin{array}{l}0 \\ 1\end{array}\right)$ is not an eigenvector of $A_{0}$ with positive eigenvalue, we can ensure that this does not lead to a twisting $n$ larger than $n_{0}$. The properties of $h$ imply that this twisting $n$, determined by

$$
2 n \pi<\sup _{t \in[-1,0]}(\varphi(t+1)-\varphi(t)) \leq 2(n+1) \pi,
$$

is equal to $n_{0}$ or $n_{0}-1$. 
The strategy in the proof of Proposition 11 is now as follows. Remove a tubular neighbourhood $T^{2} \times I$ of a torus fibre in both $\left(T_{A_{0}}, \zeta_{n_{0}}\right)$ and $\left(T_{A}, \zeta_{n}\right)$, and show that the complements are contactomorphic provided $\zeta_{n_{0}}$ corresponds to $\varphi_{0}$ and $\zeta_{n}$ to the $\varphi$ constructed in the preceding lemma. Extend this contactomorphism over a solid torus inside $T^{2} \times I$, with complement another solid torus. Finally show that the unique extensions (as tight contact structures) of the contact structures $\zeta_{n_{0}}$ resp. $\zeta_{n}$ over this last solid torus correspond to a contact (-1)surgery.

The next lemma will be essential for this final extension. Consider $B=\mathbf{R} \times$ $(\mathbf{R} / \mathbf{Z}) \times \mathbf{R}$ with coordinates $(x, y, t)$ and contact structure $\zeta^{\prime}$ given by

$$
\cos (2 \pi t) d x-\sin (2 \pi t) d y=0 .
$$

For $0<\varepsilon, \delta<1 / 4$ let

$$
V=\{(x, y, t) \in B: \delta \leq x \leq 1-\delta,-\varepsilon \leq t \leq \varepsilon\} .
$$

This will later be thought of as a tubular neighbourhood in $\left(T_{A}, \zeta_{n}\right)$ of a Legendrian circle $(t=0, x=$ const. $)$, which lies completely inside a torus fibre of $T_{A}$. Identify $\partial V$ (with corners smoothed) with $\mathbf{R}^{2} / \mathbf{Z}^{2}$ by using the standard framing of $V$. This means that the circles $y=$ const. (oriented positively in the $(t, x)$-plane) correspond to the first coordinate direction in $\mathbf{R}^{2} / \mathbf{Z}^{2}$; circles $t=$ const., $x=$ const. to the second.

Lemma 18 For every neighbourhood of $\partial V$ in $V$ (or likewise in $B \backslash V$ ), there exists a convex torus $T$ inside this neighbourhood, isotopic to $\partial V$ and satisfying $\# \Gamma_{T}=2$ and $s(T)=\infty$.

Proof The contact plane $\zeta^{\prime}$ is spanned by $\frac{\partial}{\partial t}$ and $\sin (2 \pi t) \frac{\partial}{\partial x}+\cos (2 \pi t) \frac{\partial}{\partial y}$. We may choose $T$ of the form

$$
T=\{(x, y, t) \in B:(x, t) \in \gamma\},
$$

where $\gamma$ is a smooth convex curve in the $(x, t)$-plane, close to $\partial V \cap\{y=0\}$. Moreover, we may assume that $\frac{\partial}{\partial t}$ is tangent to $\gamma$ only at the two points on $\gamma$ with $t=0$. The assumption $\varepsilon<1 / 4$ guarantees that the singular set of the characteristic foliation $\left.\zeta^{\prime}\right|_{T}$ consists of the two circles $T \cap\{t=0\}$. Furthermore, the vector spanning $\left.\zeta^{\prime}\right|_{T}$ away from its singular points always has a non-zero $\frac{\partial}{\partial x}$-component, and the coefficient functions of this vector field may be chosen not to depend on the $y$-coordinate. The two circles $\Gamma=T \cap\{x=1 / 2\}$ divide this singular foliation. Now apply Proposition 5. 
Proof of Proposition 11 (for $k=1$ ). Let $\varphi_{0}, \varphi$ be as in Lemma 17. Write $\zeta\left(\varphi_{0}\right)$ resp. $\zeta(\varphi)$ for the contact structures on $T_{A_{0}}$ resp. $T_{A}$ defined by these functions. Fix a positive real number $0<\varepsilon<1 / 2$. Let $f:[-1,0] \rightarrow[-1,0]$ be the smooth function satisfying $\varphi(f(t))=\varphi_{0}(t)$ for all $t \in[-1,0]$. Observe that $f(-1)=-1$ and $f(0)=0$, and $f$ is strictly monotone increasing.

With $B=\mathbf{R} \times(\mathbf{R} / \mathbf{Z}) \times \mathbf{R}$ as above, set

$$
B_{\sigma, \tau}=\{(x, y, t) \in B: \sigma \leq t \leq \tau\} .
$$

We continue to write $\zeta\left(\varphi_{0}\right), \zeta(\varphi)$ for the lift of those contact structures from $T_{A_{0}}$ resp. $T_{A}$ to $B$.

Define contact embeddings $F_{1}, F_{2}$ as follows:

$$
\begin{aligned}
F_{1}:\left(B_{-1+\varepsilon,-\varepsilon}, \zeta\left(\varphi_{0}\right)\right) & \longrightarrow(B, \zeta(\varphi)) \\
(x, y, t) & \longmapsto(x, y, f(t)), \\
F_{2}: \quad\left(B_{\varepsilon, 1-\varepsilon}, \zeta\left(\varphi_{0}\right)\right) & \longrightarrow(B, \zeta(\varphi)) \\
(x, y, t) & \longmapsto(x, x+y, f(t-1)+1) .
\end{aligned}
$$

Notice that $F_{2}$ is the composition of contactomorphisms

$$
(\mathbf{x}, t) \mapsto\left(A_{0}^{-1} \mathbf{x}, t-1\right) \stackrel{F_{1}}{\longmapsto}\left(A_{0}^{-1} \mathbf{x}, f(t-1)\right) \mapsto\left(A A_{0}^{-1} \mathbf{x}, f(t-1)+1\right) .
$$

Fix a positive real number $0<\delta<1 / 4$. Choose $\varepsilon>0$ sufficiently small such that

$$
\begin{gathered}
-\pi / 2<\varphi_{0}(-\varepsilon)<\varphi_{0}(\varepsilon)<\pi / 2, \\
-\pi / 2<\varphi(f(-\varepsilon))<\varphi(f(\varepsilon-1)+1)<\pi / 2,
\end{gathered}
$$

and

$$
-\delta<\tan \varphi(f(-\varepsilon))<\tan \varphi(f(\varepsilon-1)+1)<\delta .
$$

Let $g_{1}:[-\varepsilon, \varepsilon] \rightarrow \mathbf{R}$ be a smooth, strictly monotone increasing function such that

$$
g_{1}(t)= \begin{cases}f(t) & \text { for }-\varepsilon \leq t \leq-\varepsilon / 2, \\ f(t-1)+1 & \text { for } \varepsilon / 2 \leq t \leq \varepsilon \\ 0 & \text { for } t=0\end{cases}
$$

Let $g_{2}:[-\varepsilon, \varepsilon] \rightarrow \mathbf{R}$ be a smooth, monotone increasing function such that

$$
g_{2}(t)= \begin{cases}0 & \text { for }-\varepsilon \leq t \leq-\varepsilon / 2 \\ 1 & \text { for } \varepsilon / 2 \leq t \leq \varepsilon\end{cases}
$$

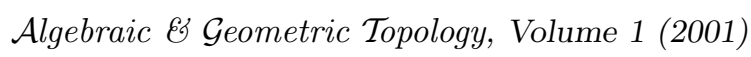


It is easy to see that $g_{2}$ can be chosen in such a way that

$$
\int_{-\varepsilon}^{\varepsilon} g_{2}^{\prime}(t) \tan \varphi\left(g_{1}(t)\right) d t=0 \text {. }
$$

For $\eta \in \mathbf{R}$ set

$$
B^{\eta}=B_{-\varepsilon, \varepsilon}^{\eta}=\{(x, y, t) \in B: x=\eta,-\varepsilon \leq t \leq \varepsilon\} .
$$

Define $h_{\eta}:[-\varepsilon, \varepsilon] \rightarrow \mathbf{R}$ by

$$
h_{\eta}(t)=\eta+\eta \int_{-\varepsilon}^{t} g_{2}^{\prime}(t) \tan \varphi\left(g_{1}(t)\right) d t .
$$

Define

$$
\begin{aligned}
\psi_{\eta}: \quad B^{\eta} & \longrightarrow B \\
(\eta, y, t) & \longmapsto\left(h_{\eta}(t), y+g_{2}(t) \eta, g_{1}(t)\right) .
\end{aligned}
$$

Notice that $\psi_{\eta}$ coincides with $F_{1}$ for $t=-\varepsilon$ and with $F_{2}$ for $t=\varepsilon$. Moreover, one easily verifies that $\psi_{\eta}$ is an injective immersion.

We compute

$$
\begin{aligned}
& \psi_{\eta}^{*}(\cos \varphi(t) d x-\sin \varphi(t) d y)= \\
& \quad=\cos \varphi\left(g_{1}(t)\right) h_{\eta}^{\prime} d t-\sin \varphi\left(g_{1}(t)\right)\left(d y+\eta g_{2}^{\prime}(t) d t\right) \\
& \quad=\sin \varphi\left(g_{1}(t)\right) d y .
\end{aligned}
$$

It follows that the singular foliation $\psi_{\eta}^{-1}\left(\left.\zeta(\varphi)\right|_{\psi_{\eta}\left(B^{\eta}\right)}\right)$ is represented by the vector field $\sin \varphi\left(g_{1}(t)\right) \frac{\partial}{\partial t}$.

The singular foliation $\left.\zeta\left(\varphi_{0}\right)\right|_{B^{\eta}}$, on the other hand, is represented by $\sin \varphi_{0}(t) \frac{\partial}{\partial t}$. We claim that these two singular foliations are identical as smooth foliations. Indeed, the two functions $s_{1}=\sin \varphi\left(g_{1}(t)\right)$ and $s_{0}=\sin \varphi_{0}(t)$ vanish only at $0 \in[-\varepsilon, \varepsilon]$ and have positive derivative there. It follows that either of them can be written as $s_{i}=t \cdot \bar{s}_{i}$ with $\bar{s}_{i}$ a smooth, nowhere zero function on $[-\varepsilon, \varepsilon]$, so $s_{1} / s_{0}$ is smooth and non-zero on all of $[-\varepsilon, \varepsilon]$.

By Proposition 6 there exists a neighbourhood $U$ of $B^{\delta} \cup B^{1-\delta}$ in $B$ and a contact embedding

$$
F:\left(U, \zeta\left(\varphi_{0}\right)\right) \longrightarrow(B, \zeta(\varphi))
$$

that coincides with $F_{1}$ resp. $F_{2}$ on the common domain of definition, and with $\psi_{\eta}$ on $B^{\eta}$ for $\eta=\delta$ or $1-\delta$.

By Proposition 3 and Lemma 18, and with $V$ as in that lemma (which holds true for the contact structure $\zeta\left(\varphi_{0}\right)$ in place of $\left.\zeta^{\prime}\right)$, this $F$ extends to a contact embedding

$$
F:\left(V_{0}, \zeta\left(\varphi_{0}\right)\right):=\left(B_{-1+\varepsilon,-\varepsilon} \cup B_{\varepsilon, 1-\varepsilon} \cup V, \zeta\left(\varphi_{0}\right)\right) \longrightarrow(B, \zeta(\varphi)) .
$$


Let $K$ be the Legendrian circle in $T_{A_{0}}$ defined by

$$
\left\{(x, y, t) \in T_{A_{0}}: x=t=0\right\} .
$$

Then $F$ induces a contact embedding

$$
\left(T_{A_{0}}-\nu K, \zeta\left(\varphi_{0}\right)\right) \longrightarrow\left(T_{A}, \zeta(\varphi)\right)
$$

where we may think of the tubular neighbourhood $\nu K$ of $K$ as

$$
\left\{(x, y, t) \in T_{A_{0}}:-\delta \leq x \leq \delta,-\varepsilon \leq t \leq \varepsilon\right\} .
$$

Again by Proposition 3 and Lemma 18 (adapted suitably), $\left(T_{A}, \zeta(\varphi)\right)$ is obtained from the manifold $\left(T_{A_{0}}, \zeta\left(\varphi_{0}\right)\right)$ by contact $(-1)$-surgery on $K$. To verify the sign of this surgery we need to make the following observations.

Let $\mu$ be a meridian of $\partial \nu K$ defined by $y=0$, say, and let $\lambda$ be a longitude of $\partial \nu K$ defined by $x=\delta, t=0$. We take $\lambda$ to be oriented in positive $y$ direction, and $\mu$ to be oriented in counterclockwise direction with respect to the oriented basis $\left(\frac{\partial}{\partial t}, \frac{\partial}{\partial x}\right)$ of the $(t, x)$-plane. This is consistent with our orientation assumptions in the definition of contact surgery. Moreover, it is this choice of longitude that gives $s(\partial \nu K)=\infty$, so the surgery coefficient $r$ is determined by expressing the attaching map in terms of $\lambda$ and $\mu$.

The effect of the map $F$ (up to isotopy) is to send $\lambda$ to $\lambda$, and $\mu$ to $\mu+\lambda$, as can be checked from our explicit formulae. So $\mu-\lambda$ maps to $\mu$, which shows that it is this curve $\mu-\lambda$ on $\partial \nu K$ which becomes homologically trivial when we glue in a solid torus in place of $\nu K$ to obtain $T_{A}$.

Acknowledgements This work was done while F.D. was visiting the Mathematical Institute of Leiden University. Part of this stay was supported by a post-doctoral fellowship from NWO (Netherlands Organisation for Scientific Research). F.D. thanks Leiden University and NWO for their support.

H.G. gratefully acknowledges support by the American Institute of Mathematics during its programme on Low-Dimensional Contact Geometry.

We also thank Ko Honda for helpful e-mail correspondence.

\section{References}

[1] Y Eliashberg, Topological characterization of Stein manifolds of dimension > 2, Internat. J. Math. 1 (1990), 29-46

[2] Y Eliashberg, Unique holomorphically fillable contact structure on the 3 -torus, Internat. Math. Res. Notices (1996) no. 2, 77-82 
[3] J B Etnyre, Symplectic convexity in low-dimensional topology, Topology Appl. 88 (1998), 3-25

[4] J B Etnyre, K Honda, Tight contact structures with no symplectic fillings, preprint, ArXiv:math.GT/0010044

[5] R Friedman, J W Morgan, Smooth Four-Manifolds and Complex Surfaces, Ergeb. Math. Grenzgeb. (3) 27, Springer (1994)

[6] E Giroux, Convexité en topologie de contact, Comment. Math. Helv. 66 (1991), 637-677

[7] E Giroux, Une structure de contact, même tendue, est plus ou moins tordue, Ann. Sci. École Norm. Sup. (4) 27 (1994), 697-705

[8] E Giroux, Une infinité de structures de contact tendues sur une infinité de variétés, Invent. Math. 135 (1999), 789-802

[9] E Giroux, Structures de contact en dimension trois et bifurcations des feuilletages de surfaces, Invent. Math. 141 (2000), 615-689

[10] R E Gompf, Handlebody construction of Stein surfaces, Ann. of Math. (2) 148 (1998), 619-693

[11] R E Gompf, A I Stipsicz, 4-Manifolds and Kirby Calculus, Grad. Stud. Math. 20, American Mathematical Society (1999)

[12] J W Gray, Some global properties of contact structures, Ann. of Math. (2) 69 (1959), 421-450

[13] K Honda, On the classification of tight contact structures I, Geom. Topol. 4 (2000), 309-368

[14] K Honda, On the classification of tight contact structures II: Torus bundles which fibre over the circle, J. Differential Geom., to appear

[15] Y Kanda, The classification of tight contact structures on the 3-torus, Comm. Anal. Geom. 5 (1997), 413-438

[16] P Libermann, C-M Marle, Symplectic Geometry and Analytical Mechanics, Math. Appl. 35, Reidel (1987)

[17] D McDuff, Symplectic manifolds with contact type boundaries, Invent. Math. 103 (1991), 651-671

[18] A Weinstein, Contact surgery and symplectic handlebodies, Hokkaido Math. J. 20 (1991), 241-251

Department of Mathematics, Peking University

Beijing 100871, P. R. China

and

Mathematisch Instituut, Universiteit Leiden

Postbus 9512, 2300 RA Leiden, Netherlands

Email: dingfan@sxx0.math.pku.edu.cn and geiges@math.leidenuniv.nl

Received: 15 December 2000 Revised: 13 February 2001 